\title{
Spät kommt Ihr, doch Ihr kommt: Warum wird die Grundrechtskonformität der Vorratsdatenspeicherungs- Richtlinie erst nach acht Jahren geklärt?
}

\author{
Thomas Giegerich* $^{*}$
}

Inhalt

I. Die Schlussanträge des Generalanwalts zur Gültigkeit der Vorratsdatenspeicherungs-Richtlinie 3

II. Beschleunigte Schaffung von Rechtsklarheit als Hauptaufgabe der Judikative 5

III. Fehlleistungen in Dublin und Luxemburg $\quad 7$

1. Geringschätzung der Grundrechtsproblematik in Klagebegründung und Streithelfervortrag des Nichtigkeitsklageverfahrens

2. Die ostentative Übergehung der Grundrechtsproblematik durch den EuGH

IV. Fehlleistung in Karlsruhe

1. Die Nichtigerklärung des deutschen Umsetzungsgesetzes durch Urteil vom 2. März 2010

2. Keine erneute Richtlinienumsetzung in Deutschland wegen Streitigkeiten innerhalb der Regierungskoalition

3. Rechtshängiges Vertragsverletzungsverfahren gegen Deutschland

4. Bestand für das Bundesverfassungsgericht wirklich kein Anlass zur Kooperation mit dem EuGH im Vorabentscheidungsverfahren?

\section{Die Schlussanträge des Generalanwalts zur Gültigkeit der Vorratsdatenspeicherungs-Richtlinie}

Am 12. Dezember 2013 hat Generalanwalt Cruz Villalón dem Gerichtshof der EU folgende Entscheidungen vorgeschlagen: Erstens die Ungültigkeit der Richtlinie 2006/24/EG vom 15. März 2006 über die Vorratsspeicherung von Daten, die bei der Bereitstellung öffentlich zugänglicher elektronischer Kommunikationsdienste oder öffentlicher Kommunikationsnetze erzeugt und verarbeitet werden, ${ }^{1}$ festzustellen; ${ }^{2}$ zweitens die Wirkung dieser Feststellung auszusetzen, bis der Unionsgesetzgeber innerhalb angemessener Frist die Maß-

* Prof. Dr. Thomas Giegerich, LL.M. (University of Virginia), Jean-Monnet-Lehrstuhl für Europarecht und Europäische Integration und Direktor des Europa-Instituts (Sektion Rechtswissenschaft), Universität des Saarlandes.

1 ABl. L 105 v. 13.4.2006, S. 54.

2 Siehe zur genauen Formulierung noch unten Text bei Fn. $11 \mathrm{f}$. 
nahmen ergreift, die erforderlich sind, um der festgestellten Ungültigkeit abzuhelfen. ${ }^{3}$ Diesen Schlussanträgen liegen zwei Vorabentscheidungsersuchen des High Court of Ireland und des österreichischen Verfassungsgerichtshofs aus dem Jahr 2012 zugrunde.

Nach Auffassung des Generalanwalts verstößt die Richtlinie in zweifacher Hinsicht gegen die Charta der Grundrechte der EU (EUGrCh). Zum einen hält er sie für unvereinbar mit Art. 52 Abs. 1 EUGrCh, weil sie den Anforderungen an die inhaltliche Bestimmtheit von grundrechtseinschränkenden Gesetzen nicht genüge. Zum anderen wirft der Generalanwalt dem Unionsgesetzgeber einen Verstoß gegen das Verhältnismäßigkeitsprinzip im Sinne von Art. 52 Abs. 1 EUGrCh vor, weil er die höchstzulässige Dauer der Datenspeicherung in Art. 6 der Richtlinie auf zwei Jahren festgelegt habe, obwohl kein überzeugender Grund für eine längere Dauer als ein Jahr erkennbar sei. ${ }^{4}$

Seine Anforderungen an die inhaltliche Bestimmtheit der Richtlinie entnimmt der Generalanwalt dem Tatbestandsmerkmal ,gesetzlich vorgesehen“ in Art. 52 Abs. 1 Satz 1 EUGrCh. ${ }^{5}$ Er lehnt sich dabei an die einschlägige Rechtsprechung des Europäischen Gerichtshofs für Menschenrechte zu den entsprechenden Einschränkungsklauseln der EMRK an. ${ }^{6}$ Er weist überdies darauf hin, dass sich entsprechende Anforderungen aus dem Verhältnismäßigkeitserfordernis in Art. 52 Abs. 1 Satz 2 EUGrCh ergäben. ${ }^{7}$ Artikel 52 Abs. 1 EUGrCh verlange vom Unionsgesetzgeber, wenn er selbst qualifizierte Grundrechtseingriffe durch die Mitgliedstaaten anordne (wie hier Eingriffe in die Grundrechte auf Privatleben und Schutz personenbezogener Daten gemäß Art. 7 und Art. 8 EUGrCh), Schutzmaßnahmen, um ,die den Mitgliedstaaten übertragene sehr weite Befugnis hinsichtlich des Zugangs zu den Daten und deren Auswertung durch den Erlass von Spezifikationen in Form von Prinzipien grundsätzlich einzugrenzen“" ${ }^{8}$ Der Unionsgesetzgeber dürfe die Begrenzung solcher Grundrechtseingriffe nicht ganz den mitgliedstaatlichen Legislativen, Verwaltungsbehörden und/oder Justizorganen überlassen, weil sonst seiner eigenen Grundrechtsbindung nach Art. 51 Abs. 1 EUGrCh ihr Sinn genommen würde. In der Tat zeigt die Textfassung der genannten Bestimmung, dass die Grundrechtsbindung des EU-Gesetzgebers derjenigen der Mitgliedstaaten gegenüber vorrangig ist.

Der Generalanwalt nennt beispielhaft folgende Vorgaben, die den mitgliedstaatlichen Gesetzgebern hätten gemacht werden müssen: eine hinreichend präzise Umschreibung der Straftatbestände, die den Zugang zu den infolge der Richtlinie angelegten Datenvorräten rechtfertigen können; die Monopolisierung der Zugangsentscheidung bei den Justizbehörden oder sonstigen unabhängigen Stellen; Vorkehrungen für außergewöhnliche Fälle (z.B. in Bezug auf Daten, die der ärztlichen Schweigepflicht unterliegen); die Pflicht zur Löschung nicht mehr benötigter Daten bzw. die Unterrichtung der Betroffenen über den er-

3 Schlussanträge GA Cruz Villalón zu EuGH, verb. Rs. C-293/12 und C-594/12, Digital Rights Ireland.

4 Ibid., Nr. $140 \mathrm{ff}$.

5 Ibid., Nr. $108 \mathrm{ff}$.

6 Diese Anlehnung an die Straßburger Rechtsprechung wird auf die Kohärenzklausel in Art. 52 Abs. 3 EUGrCh gestützt.

7 Schlussanträge GA Cruz Villalón zu EuGH, verb. Rs. C-293/12 und C-594/12, Digital Rights Ireland, Nr. $133 \mathrm{ff}$.

8 Ibid., Nrn. 118, 120. 
folgten Zugang, sobald dies ohne Vereitelung des Zugangszwecks möglich sei. ${ }^{9}$ Statt seiner eigenen grundrechtlichen Schutzverantwortung gerecht zu werden, habe sich der Unionsgesetzgeber in dieser Hinsicht in Art. 4 der Richtlinie zu weitgehend auf die Mitgliedstaaten verlassen.

Der Generalanwalt problematisiert nicht, ob die Harmonisierungskompetenz für den Binnenmarkt (Art. 114 AEUV), auf der die Richtlinie beruht, ${ }^{10}$ eine ausreichende Ermächtigungsgrundlage auch für die von ihm geforderten Vorgaben darstellt. Er sieht dies offenbar deshalb als selbstverständlich an, weil die den Mitgliedstaaten von der Richtlinie auferlegte Grundrechtseingriffspflicht durch derartige Regelungen abgeschwächt würde. Man könnte hier an eine Art die Individualfreiheit wahrende (aber die Souveränität der Mitgliedstaaten begrenzende) Annexkompetenz zur Sicherung der Verhältnismäßigkeit von EU-rechtlich vorgeschriebenen Grundrechtseingriffen denken. Diese an sich schlüssige Herleitung der EU-Kompetenz wird jedoch wahrscheinlich im Lager derjenigen auf Widerspruch stoßen, denen Art. 114 AEUV von vornherein als unzureichende Ermächtigung für die Vorratsdatenspeicherung erschien.

Im Ergebnis hält der Generalanwalt die Richtlinie zwar in vollem Umfang für grundrechtswidrig, doch sind die ermittelten Verstöße seiner Ansicht nach durch gesetzgeberische Nachbesserungen ohne große Schwierigkeiten zu bereinigen. Deshalb schlägt er dem Gerichtshof auch vor, den Gesetzgebungsakt nicht für nichtig zu erklären, sondern nur, ihn für ungültig zu erklären bzw. seine Ungültigkeit festzustellen bzw. - so die Formulierung der letztendlich vorgeschlagenen Antworten an die vorlegenden Gerichte - seine Unvereinbarkeit mit der EU-Grundrechtecharta festzustellen. ${ }^{11}$ Anscheinend war der Generalanwalt sich selbst nicht ganz im Klaren darüber, was genau der EuGH entscheiden sollte. ${ }^{12}$ Jedenfalls hält er es aufgrund des im Vorabentscheidungsverfahren analog anwendbaren Art. 264 Abs. 2 AEUV im Interesse der Rechtssicherheit für geboten, die „Wirkungen der Feststellung der Ungültigkeit“ für eine angemessene Frist auszusetzen, damit der Unionsgesetzgeber die notwendigen Reparaturen vornehmen kann. ${ }^{13}$ Denn der Generalanwalt erkennt die Dringlichkeit der Endziele der von der Richtlinie verlangten Grundrechtseingriffe (d.h. die Verfügbarkeit des angelegten Datenvorrats zwecks Ermittlung und Verfolgung schwerer Straftaten) an.

\section{Beschleunigte Schaffung von Rechtsklarheit als Hauptaufgabe der Judikative}

Die Schlussanträge sind überzeugend begründet. Es bleibt freilich abzuwarten, ob der EuGH ihnen in der dogmatischen Konstruktion oder zumindest im Ergebnis folgen wird.

9 Ibid., Nr. $125 \mathrm{ff}$.

10 Vgl. zu ihrer Tragfähigkeit die in Fn. 28 nachgewiesene EuGH-Entscheidung.

11 Schlussanträge GA Cruz Villalón zu EuGH, verb. Rs. C-293/12 und C-594/12, Digital Rights Ireland, Nrn. 154, 155, 159.

12 Vgl. die Unterscheidung zwischen der Nichtigerklärung und der Feststellung der Unvereinbarkeit mit dem Grundgesetz bei Gesetzen im deutschen Verfassungsprozessrecht: Klein, in: Benda/ Klein/Klein, Verfassungsprozessrecht, 3. Aufl. 2012, § 39, Rdnr. $1392 \mathrm{ff}$.

13 Schlussanträge GA Cruz Villalón zu EuGH, verb. Rs. C-293/12 und C-594/12, Digital Rights Ireland, $\mathrm{Nr} .158$. 
Die Herstellung einer angemessenen Balance zwischen Freiheitswahrung und wirksamer Bekämpfung terroristischer und anderer schwerer Kriminalität stellt zweifelsohne eine große Herausforderung dar. Angesichts der intensiven und teilweise erregten öffentlichen Debatte über den grenzüberschreitenden Datenschutz im Zusammenhang mit den Enthüllungen des US-amerikanischen Whistleblowers Edward Snowden ist zu erwarten, dass der Gerichtshof dem Schutz der Privatsphäre einen hohen Stellenwert einräumen wird, vielleicht sogar einen größeren als der Generalanwalt. Zumindest wird er die Grundrechtskonformität der Vorratsdatenspeicherungs-Richtlinie in der einen oder anderen Weise ein für alle Mal klären.

Mir geht es vorliegend aber nicht um eine inhaltliche, sondern um eine zeitliche Kritik: Wie kommt es, dass eine Frage, die in der Öffentlichkeit seit dem Erlass der Richtlinie umstritten war, ${ }^{14}$ erst jetzt - acht Jahre später - vom EuGH definitiv geklärt wird? Eine derartige Säumnis ist im vorliegenden Fall rechtsstaatlich besonders schwer erträglich, weil die Vorratsdatenspeicherungs-Richtlinie täglich zu vieltausendfachen Eingriffen in die Privatsphäre führt, denen wir alle unausweichlich ausgesetzt sind und die von erheblichen Teilen der Unionsbürgerschaft seit langem für grundrechtswidrig gehalten werden. Ob dies zu Recht oder zu Unrecht geschieht, darf nicht jahrelang in der Schwebe bleiben, sonst leidet das Vertrauen in die Rechtsstaatlichkeit sowohl der EU als auch der Mitgliedstaaten. Nicht zuletzt grundrechtliche Bedenken haben dazu geführt, dass etliche Mitgliedstaaten, darunter Deutschland, die Richtlinie nicht oder nur verspätet in nationales Recht umgesetzt haben.

Für diese Verzögerung im gerichtlichen Klärungsprozess verantwortlich sind drei Fehlentscheidungen zunächst der irischen Regierung, dann des EuGH und schließlich des Bundesverfassungsgerichts, die sich als verpasste Gelegenheiten zur zügigeren Klärung der Grundrechtsproblematik erweisen. Besonders gravierend sind dabei die Luxemburger und Karlsruher Fehlleistungen. Denn die Hauptaufgabe der Judikative besteht darin, im Rahmen ihrer Zuständigkeit das Recht zu wahren, ${ }^{15}$ Rechtsklarheit zu schaffen und dadurch Rechtsfrieden zu stiften. ${ }^{16}$ Diese Aufgabe verlangt es der rechtsprechenden Gewalt auch im europäischen Mehrebenensystem ab, in einem zulässigerweise gegen einen Gesetzgebungsakt anhängig gemachten Verfahren naheliegende Gültigkeitseinwände - insbesondere solche grundrechtlicher Art - nicht unbeantwortet zu lassen. Sie darf sich ihrer Entscheidungsverantwortung nicht durch Kunstgriffe, etwa eine übermäßige Engführung der eigenen Kompetenz oder einen vorschnellen Rückgriff auf Doktrinen richterlicher Enthaltsamkeit, ${ }^{17}$ entziehen, weder im Interesse der Arbeitsökonomie noch zwecks Vermei-

14 Vgl. die Nachweise in BVerfGE 125, 160 (Rdnr. 82 der unter www.bverfg.de abrufbaren elektronischen Fassung).

15 Vgl. für den EuGH ausdrücklich Art. 19 Abs. 1 Satz 2 EUV. Für das BVerfG ergibt sich Entsprechendes implizit aus Art. 92 GG, der ihm rechtsprechende Gewalt „,anvertraut“, spezifisch jedoch die Wahrung der Verfassung - vgl. Benda/Klein, in: Benda/Klein/Klein, (Fn. 12), §1, Rdnr. 17. Berühmt ist die Aussage von Chief Justice Marshall in Marbury v. Madison, 5 U.S. (1 Cranch) 137, 177 (1803): ,[I]t is emphatically the province and duty of the Judicial Department to say what the law is."

16 Auf dieser Grundlage beruht etwa § 78 Satz 2 BVerfGG, näher Klein, (Fn. 12), § 22, Rdnr. 710 ff.

17 Z.B. die aus den USA stammende Political-Question-Doktrin, näher Henkin, Foreign Affairs and the US Constitution, 2. Aufl. 1996, S. $143 \mathrm{ff}$. 
dung politisch heikler Rechtsfragen. Im Gegenteil muss diejenige vertretbare Auslegung von Verfahrensvorschriften Vorrang genießen, die es dem erkennenden Gericht ermöglicht, seine Rechtswahrungs- und Rechtsklärungsaufgabe im Interesse des Rechtsfriedens effektiv und schnellstmöglich zu erfüllen. Auch in dieser Hinsicht gilt: Wer bald gibt, gibt doppelt.

Im europäischen Mehrebenensystem sind mitgliedstaatliche und europäische Gerichte gehalten, zusammenzuwirken zur beschleunigten Klärung der Frage, ob ein EU-Sekundärrechtsakt mit den europäischen Grundrechten vereinbar ist. Jede der beiden Gerichtsebenen prüft diese Frage in jedem einschlägigen Fall für sich, und gegebenenfalls richtet das nationale Gericht ein sorgfältig ausgearbeitetes Vorlageersuchen an den EuGH.

\section{Fehlleistungen in Dublin und Luxemburg}

\section{Geringschätzung der Grundrechtsproblematik in Klagebegründung und Streithelfervortrag des Nichtigkeitsklageverfahrens}

Die Dubliner Fehlleistung hängt mit der erfolglos gebliebenen Nichtigkeitsklage zusammen, welche Irland, unterstützt durch die Slowakei, bereits im Juli 2006 gegen die Vorratsdatenspeicherungs-Richtlinie erhoben hatte. ${ }^{18}$ Diese beiden Staaten hatten im Rat als einzige gegen die Richtlinie gestimmt. ${ }^{19}$ Irland stützte seine alsbald erhobene Klage damals allein darauf, dass die Richtlinie nicht auf einer geeigneten Rechtsgrundlage erlassen worden sei. Die herangezogene Binnenmarkt-Harmonisierungskompetenz ${ }^{20}$ könne keine Richtlinie tragen, deren Hauptzweck die Ermittlung, Feststellung und Verfolgung von Straftaten sei. ${ }^{21}$ Vielmehr hätte diese (als Rahmenbeschluss) auf Art. 30, Art. 31 Abs. 1 lit. c und Art. 34 Abs. 2 lit. b EUV damaliger Fassung gestützt werden müssen. Einen Grundrechtsverstoß rügte Irland hingegen nicht, obwohl er nahelag. War die Sensibilität für den Datenschutz in Irland unterentwickelt? Aus steuerlichen Gründen haben in Irland seit längerem zahlreiche IT- und Internetkonzerne wie Google ihren europäischen Sitz, die an strengen Datenschutzregeln nicht interessiert sind. Wollte sich die irische Regierung deshalb nicht für ein europäisches Grundrecht auf Datenschutz einsetzen?

Die Slowakei als Streithelferin machte immerhin ergänzend geltend, die „Vorratsspeicherung von personenbezogenen Daten in dem nach der Richtlinie 2006/24 verlangten Umfang führe zu einem erheblichen Eingriff in das Recht Einzelner auf Achtung ihres Privatlebens nach Art. 8 EMRK. Es sei zweifelhaft, ob ein so bedeutender Eingriff aus wirtschaftlichen Gründen, im vorliegenden Fall wegen des besseren Funktionierens des

18 EuGH, Rs. C-301/06, Irland/Europäisches Parlament und Rat der Europäischen Union, Slg. 2009, I-593.

19 Schlussanträge GA Bot zu EuGH, Rs. C-301/06, Irland/Europäisches Parlament und Rat der Europäischen Union, Slg. 2009, I-593, Nr. 35.

20 Art. 95 EG-Vertrag, nach Änderung jetzt Art. 114 AEUV.

21 Bei Erlass der Richtlinie im Jahr 2006 war die Polizeiliche und Justizielle Zusammenarbeit in Strafsachen noch in der intergouvernemental strukturierten 3. Säule der EU außerhalb des eigentlichen Integrationsbereichs angesiedelt. 
Binnenmarkts, gerechtfertigt werden könne." ${ }^{22}$ Auch das Europäische Parlament und der Rat gingen in ihrer Verteidigung auf Art. 8 EMRK ein und trugen in Anlehnung an die Begründungserwägung 9 der Richtlinie vor, dass der darin vorgesehene Grundrechtseingriff wegen überwiegender öffentlicher Interessen gerechtfertigt sei. ${ }^{23}$ Die Bezugnahme auf Art. 8 EMRK anstelle von Art. 7 und 8 EUGrCh erklärt sich damit, dass die Charta erst nach dem Inkrafttreten des Vertrags von Lissabon am 1. Dezember 2009 Primärrechtsrang erhielt. ${ }^{24}$ Demgegenüber war die EMRK schon lange als Erkenntnisquelle bei der richterlichen Entwicklung von ungeschriebenen Grundrechten als allgemeinen Rechtsgrundsätzen des Primärrechts anerkannt. ${ }^{25}$

Damit war die ohnedies auf der Hand liegende Grundrechtsproblematik neben der Frage der passenden Ermächtigungsgrundlage eigentlich hinreichend deutlich ins Verfahren eingeführt worden, wenngleich sie offenbar von keinem der Beteiligten wirklich ernstgenommen wurde. Dies zeigt sich unter anderem daran, dass die Slowakei (im Gegensatz zu Irland) dem EuGH vorschlug, im Falle der Nichtigerklärung der Richtlinie die Wirkungen des Urteils bis zur Annahme eines ersetzenden Rechtsakts auszusetzen. ${ }^{26}$ Auch sie nahm offenbar an, dass sich die Vorratsdatenspeicherung allein dadurch problemlos grundrechtskonform ausgestalten ließe, dass sie auf eine andere Rechtsgrundlage gestützt würde. Dementsprechend ging der Generalanwalt in seinen Schlussanträgen mehr als zwei Jahre nach der Klageerhebung nur am Rande auf Art. 8 EMRK ein und ließ dabei erkennen, dass er den diesbezüglichen Eingriff für gerechtfertigt hielt. ${ }^{27}$

\section{Die ostentative Übergehung der Grundrechtsproblematik durch den EuGH}

Die Große Kammer des EuGH stellte am Anfang ihrer rechtlichen Würdigung im Urteil vom 10. Februar 2009 - kurz vor dem dritten Geburtstag der Richtlinie - klar, „dass sich die von Irland erhobene Klage allein auf die Wahl der Rechtsgrundlage bezieht und nicht auf eine eventuelle Verletzung der Grundrechte als Folge von mit der Richtlinie 2006/24 verbundenen Eingriffen in das Recht auf Privatsphäre. " ${ }^{28} \mathrm{Im}$ Ergebnis wies der Gerichtshof die Nichtigkeitsklage ab, weil er die im Begründungsvorspann der Richtlinie angegebene Rechtsgrundlage für korrekt hielt. Warum verweigerte der EuGH die Grundrechtsprüfung und ließ die diesbezügliche Rechtsunsicherheit bestehen? Denn offenbar sah man - anders als der Generalanwalt - durchaus Grundrechtsprobleme, sonst wäre die erwähnte Klarstellung unterblieben. Dementsprechend hatte schon mehr als achtzehn Monate zuvor die Ge-

22 EuGH, Rs. C-301/06, Irland/Europäisches Parlament und Rat der Europäischen Union, Slg. 2009, I-593, Rdnr. 34.

23 Ibid., Rdnrn. 39, 46.

24 Art. 6 Abs. 1 EUV n.F.

25 Art. 6 Abs. 2 a.F. (Abs. 3 n.F.) EUV.

26 Schlussanträge GA Bot zu EuGH, Rs. C-301/06, Irland/Europäisches Parlament und Rat der Europäischen Union, Slg. 2009, I-593, Nr. 51.

27 Ibid., Nr. $94 \mathrm{f}$.

28 EuGH, Rs. C-301/06, Irland/Europäisches Parlament und Rat der Europäischen Union, Slg. 2009, I-593, Rdnr. 57. Kritisch Simitis, Der EuGH und die Vorratsdatenspeicherung oder die verfehlte Kehrtwende bei der Kompetenzregelung, NJW 2009, S. 1782 ff.; Bernsdorff, in: Meyer (Hrsg.), Charta der Grundrechte der Europäischen Union, 3. Aufl. 2011, Art. 8, Rdnr. 23 b. 
neralanwältin Kokott in ihren Schlussanträgen in einem anderen Verfahren Folgendes ausgeführt: „Man kann daran zweifeln, ob die Speicherung von Verkehrsdaten aller Nutzer - gewissermaßen auf Vorrat - mit Grundrechten vereinbar ist, insbesondere da dies ohne konkreten Verdacht geschieht. [...] Ein den Zweifeln entsprechender Durchgriff auf die Grundrechte würde den Rahmen des Verfahrens sprengen [...] Möglicherweise ist diese Frage eines Tages aus Anlass der Richtlinie 2006/24 zu prüfen, die eine gemeinschaftsrechtliche Verpflichtung zur Vorratsspeicherung einführt." 29

Die vorgenannte Klarstellung des EuGH erfolgte zu einer Zeit, als die Frist zur Erhebung von Nichtigkeitsklagen gegen die Vorratsdatenspeicherungs-Richtlinie nach Art. 263 Abs. 6 AEUV längst abgelaufen war. $\mathrm{Da}$ - von Irland und der Slowakei abgesehen - alle übrigen nach Art. 263 Abs. 2 AEUV Klageberechtigten diese Richtlinie gutgeheißen hatten, war eine weitere Nichtigkeitsklage ohnedies von vornherein praktisch ausgeschlossen. Die Klagemöglichkeit nationaler Parlamente bzw. ihrer Kammern nach Art. 8 des Protokolls über die Anwendung der Grundsätze der Subsidiarität und der Verhältnismäßigkeit $^{30}$ wurde erst durch den Vertrag von Lissabon eingeführt und dient außerdem allein zur Durchsetzung des Subsidiaritätsprinzips, nicht der Grundrechte. Die Klarstellung des EuGH musste infolgedessen geradezu als Einladung an die nationalen Gerichte verstanden werden, ihm die Frage der Vereinbarkeit der Richtlinie mit den Grundrechten im Wege des Vorabentscheidungsersuchens nach Art. 267 AEUV zu unterbreiten.

Wieso aber wählte der EuGH diesen Umweg? Im Verfahren der Nichtigkeitsklage gegen Sekundärrechtsakte prüft der Gerichtshof nicht von Amts wegen alle in Frage kommenden Nichtigkeitsgründe, sondern beschränkt sich grundsätzlich auf diejenigen, die der Kläger zumindest der Sache nach in seinen Vortrag aufgenommen hat. ${ }^{31}$ Im Einklang damit ist das nachträgliche Vorbringen neuer Klagegründe unzulässig, wenn diese nicht auf erst während des Verfahrens zutage getretene rechtliche oder tatsächliche Gesichtspunkte gestützt werden. ${ }^{32}$ Dies soll Verfahrensverzögerungen durch ,scheibchenweises“ Klagevorbringen unterbinden. Der EuGH behält sich jedoch vor, jedenfalls bestimmte Nichtigkeitsgründe von Amts wegen zu prüfen, ohne dass seine Praxis einheitlich und damit sicher vorhersehbar wäre. ${ }^{33}$

Im vorliegenden Fall war der EuGH aber nicht bereit, von Amts wegen über die Grundrechtswidrigkeit der Vorratsdatenspeicherungs-Richtlinie zu entscheiden. Zwar hatte die Slowakei als Streithelferin die Grundrechtsfrage (halbherzig) in das Verfahren eingeführt. Indessen billigt der Gerichtshof Streithelfern im Hinblick auf die Akzessorietät der Streithilfe nach Art. 129 EuGH-VerfO nur eine begrenzte Funktion in dem Sinne zu, dass sie keine von der Hauptpartei nicht angeführten Nichtigkeitsgründe - zum Beispiel Grund-

29 Schlussanträge GAin Kokott zu EuGH, Rs. C-275/06, Promusicae, Slg. 2008, I-271, Nr. 82 (Fußnoten weggelassen). Zu diesem Zeitpunkt war die Klage Irlands gegen die Vorratsdatenspeicherungs-Richtlinie bereits anhängig.

30 In der Fassung v. 13.12.2007, ABl. C 326 v. 26.10.2012, S. 206.

31 Pechstein, EU-Prozessrecht, 4. Aufl. 2011, Rdnrn. 524, 543. Vgl. Art. 120 lit. c der EuGH-VerfO v. 25.9.2012 (Sartorius II, Nr. 250), der eine Entsprechung in der Vorgängerfassung der EuGHVerfO von 1991 hatte.

32 Art. 127 Abs. 1 EuGH-Verfo.

33 Zur Kritik Pechstein, (Fn. 31), Rdnr. 524 m.w.N. 
rechtsverletzungen - geltend machen können. ${ }^{34}$ Diese richterliche Zurückhaltung ist nicht etwa der Funktion der Nichtigkeitsklage oder der Streithilfe geschuldet, wenn Mitgliedstaaten als Kläger oder Streithelfer fungieren, denn deren Prozessführung erfolgt im objektiven Interesse an rechtmäßigen Verhältnissen in der EU und nicht zur Durchsetzung subjektiver Rechte. Vielmehr beruht sie auf einer übertrieben formalistischen prozessualen Rollenverteilung, die vielleicht die Verfahren beschleunigen und die Arbeitslast des EuGH vermindern mag, der Gerechtigkeit und dem Rechtsfrieden aber abträglich ist.

In Vorabentscheidungsverfahren über Gültigkeitsfragen nach Art. 267 Abs. 1 lit.b AEUV beschränkt der EuGH sich übrigens ganz entsprechend auf die vom vorlegenden Gericht in Bezug genommenen Ungültigkeitsgründe. ${ }^{35}$ Auch hier geht es dem Gerichtshof offenbar vorrangig um schnelle Verfahrenserledigungen. Dies steht indessen in einem Spannungsverhältnis zu seiner Praxis in Vorabentscheidungsverfahren zur Auslegung von Normen des EU-Rechts, in denen er Vorlagefragen durchaus umformuliert oder dem nationalen Gericht über dessen Fragen hinausgehende Hilfestellungen leistet, damit es die Vereinbarkeit nationaler Vorschriften mit dem EU-Recht vernünftig beurteilen kann. ${ }^{36}$

Das vorliegende Verfahren zeigt in aller Deutlichkeit, dass die EuGH-Praxis der zurückhaltenden Normenkontrolle sowohl im Nichtigkeitsklage- als auch im Vorabentscheidungsverfahren überdacht werden muss. Dies gilt zumindest insoweit, als die Vereinbarkeit eines Sekundärrechtsakts mit den Grundrechten - wie hier - offenkundig problematisch ist. Diese Problematik muss im Interesse der menschenrechtlichen Glaubwürdigkeit der EU umgehend geklärt werden. Denn in einer Union, welche die Wahrung der Menschenrechte gemäß Art. 2 EUV zu ihren Grundwerten zählt und diese der Außenwelt (einschließlich dem UN-Sicherheitsrat) gegenüber geradezu offensiv vertritt, ${ }^{37}$ gehört deren entschlossene Durchsetzung nach innen zu den unabdingbaren Aufgaben der Unionsgerichtsbarkeit. Dies gilt zumal, seit die EU über einen zunächst im Jahr 2000 feierlich proklamierten und dann 2009 durch den Vertrag von Lissabon konstitutionalisierten umfassenden Grundrechtskatalog verfügt. Zwar erweitert die EU-Grundrechtecharta nicht die Zuständigkeiten der Union im Allgemeinen ${ }^{38}$ und des EuGH im Besonderen. ${ }^{39}$ Wenn der EuGH jedoch als Grundrechtsschutzinstanz innerhalb und außerhalb der EU ernstgenommen werden will, muss er seine vorhandenen Zuständigkeiten grundrechtsfreundlich handhaben. Dazu gehört es, im Rahmen eines zulässigerweise bei ihm anhängig gemachten Normenkontrollverfahrens die Grundrechtmäßigkeit eines Sekundärrechtsakts bei ernstlichen Zweifeln notfalls von Amts wegen zu prüfen. Eine so verstandene Grundrechtssensibilität und -verantwortung muss der EuGH auch deshalb dokumentieren, weil sie letztlich

34 Dittert, Die neue Verfahrensordnung des EuGH, EuZW 2013, S. 729 f. unter Hinweis auf EuGH, Rs. C-155/91, Kommission/Rat, Slg. 1993, I-963, Rdnr. 24; Schlussanträge GAin Kokott zu EuGH, Rs. C-550/07 P, Akzo Nobel Chemicals, Slg. 2010, I-8301, Nr. 156.

35 Vgl. z.B. EuGH, Rs. C-26/96, Rotexchemie, Slg. 1997, I-2817, Rdnr. 6 - entgegen den Schlussanträgen GA Colomer, Nr. 13 f.; EuGH, Rs. C-408/95, Eurotunnel, Slg. 1997, I-6315, Rdnr. 34.

36 Wegener, in: Calliess/Ruffert (Hrsg.), EUV/AEUV, 4. Aufl. 2011, Art. 267 AEUV, Rdnr. 5 f.

37 Art. 21 Abs. 1, Abs. 2 lit. b EUV. EuGH, verb. Rs. C-402/05 P und C-415/05 P, Kadi I, Slg. 2008, I-6351, Rdnr. 280 ff.; EuGH, verb. Rs. C-584/10 P, C-593/10 P und C-595/10 P, Kadi II, Slg. 2013, Rdnr. $97 \mathrm{ff}$.

38 Art. 6 Abs. 1 UAbs. 2 EUV.

39 Art. 51 Abs. 1 Satz 2, Abs. 2 EUGrCh. 
ausgesprochene oder unausgesprochene Bedingung dafür ist, dass der Vorrang des EUSekundärrechts vor den nationalen Grundrechten in den Mitgliedstaaten akzeptiert wird.

Eine solchermaßen erweiterte grundrechtliche Kontrollbereitschaft des EuGH wird noch wichtiger werden, sobald die EU nach Art. 6 Abs. 2 EUV der EMRK beigetreten ist. Ansonsten würde die nachgeschaltete Menschenrechtskontrolle durch den Europäischen Gerichtshof für Menschenrechte unnötig verzögert, weil nach dem vorliegenden revidierten Entwurf eines Abkommens über den Beitritt der EU zur EMRK vom 10. Juni $2013^{40}$ eine Vorabbefassung des EuGH mit der Grundrechtsfrage notfalls durch Unterbrechung des Straßburger Verfahrens sichergestellt werden muss. ${ }^{41}$ Wenn es also die nationalen Gerichte versäumt haben, den EuGH einzuschalten, muss der EGMR dies nachholen.

In grundrechtlicher Perspektive hat die Große Kammer des EuGH nach alledem mit ihrer bloßen Klarstellung sehenden Auges eine bedauerliche Fehlleistung produziert.

\section{Fehlleistung in Karlsruhe}

\section{Die Nichtigerklärung des deutschen Umsetzungsgesetzes durch Urteil vom 2. März 2010}

Auch das Bundesverfassungsgericht war schon vor Jahren mit der Vorratsdatenspeicherungs-Richtlinie befasst, ohne deren Vereinbarkeit mit den europäischen Grundrechten einer Klärung näherzubringen. Darin liegt letztendlich eine ebenso bedauerliche Fehlleistung. Etwa 34.000 Verfassungsbeschwerden gegen das leicht verspätete deutsche Umsetzungsgesetz, ${ }^{42}$ das aus der Zeit der CDU/CSU-SPD-Koalition (seit 2005) stammte, die auch die Richtlinie mitgetragen hatte, waren seit 2008 in Karlsruhe anhängig und wurden ein gutes Jahr nach dem EuGH mit Urteil vom 2. März 2010 entschieden. ${ }^{43}$ Eine der Beschwerdeführerinnen war die spätere Bundesjustizministerin der CDU/CSU-FDP-Koalition (seit 2009), Leutheusser-Schnarrenberger (FDP), der nach ihrem Amtsantritt die Aufgabe zuwachsen sollte, für die korrekte Umsetzung der Richtlinie in Deutschland zu sorgen.

Die Verfassungsbeschwerden waren erfolgreich: Das Bundesverfassungsgericht erklärte eine Reihe von deutschen Gesetzesvorschriften im Telekommunikationsgesetz und in der Strafprozessordnung wegen Verstoßes gegen Art. 10 Abs. 1 GG für ganz oder teilweise nichtig. Es hielt die Vorratsdatenspeicherung zwar nicht von vornherein für unverhältnis-

40 Fifth Negotiation Meeting between the CDDH Ad Hoc Negotiation Group and the European Commission on the Accession of the European Union to the European Convention on Human Rights: Final Report to the CDDH, Appendix I, 10 June 2013, Dokument 47+1(2013)008rev 2, abgedruckt in: Europa-Institut of Saarland University (ed.), European Law - Selected Documents, 2013, S. $423 \mathrm{ff}$.

41 Art. 3 Abs. 6 des Übereinkommens-Entwurfs. Näher Polakiewicz, Der Abkommensentwurf über den Beitritt der Europäischen Union zur Europäischen Menschenrechtskonvention, EuGRZ 2013, S. $478 \mathrm{f}$.

42 Gesetz zur Neuregelung der Telekommunikationsüberwachung und anderer verdeckter Ermittlungsmaßnahmen sowie zur Umsetzung der Richtlinie 2006/24/EG vom 21.12.2007, BGB1. I 2007, 3198, in Kraft seit dem 1.1.2008. Die Umsetzungsfrist war bereits am 15.9.2007 abgelaufen.

43 BVerfGE 125, 260 (1 BvR 256/08 u.a.) - hier zitiert nach der elektronischen Fassung (abrufbar unter www.bverfg.de). 
mäßig, entnahm dem Grundgesetz jedoch hohe Anforderungen ,insbesondere hinsichtlich der Datensicherheit, des Umfangs der Datenverwendung, der Transparenz und des Rechtschutzes. Nur wenn diesbezüglich hinreichend anspruchsvolle und normenklare Regelungen getroffen sind, ist der in einer solchen Speicherung liegende Eingriff verhältnismäßig

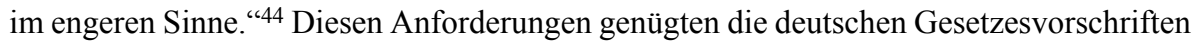
nach Auffassung der Senatsmehrheit nicht. Sie entsprechen übrigens weitgehend den Anforderungen, denen nach den eingangs dargestellten Schlussanträgen des Generalanwalts Cruz Villalón die Richtlinie selbst in dem Sinne zu genügen hat, dass sie den nationalen Gesetzgebern als Vorgaben bei der Umsetzung auferlegt werden.

Das Bundesverfassungsgericht hatte die Anwendung der betreffenden deutschen Gesetzesvorschriften bereits zuvor durch einstweilige Anordnung eingeschränkt ${ }^{45}$ und diese Einschränkungen später erweitert und verlängert. ${ }^{46}$ Dabei hatte es seine Einschränkungen so sorgsam formuliert, dass die Erfüllung der europarechtlichen Verpflichtungen Deutschlands aus der Vorratsdatenspeicherungs-Richtlinie weiterhin sichergestellt war. In der Hauptsacheentscheidung vom 2. März 2010 nahm das Gericht darauf aber keinerlei Rücksicht mehr. Mit der Nichtigerklärung der deutschen Umsetzungsbestimmungen verstieß Deutschland fortlaufend gegen seine unbestreitbare Durchführungspflicht nach Art. 291 Abs. 1 AEUV in Bezug auf die Richtlinie.

\section{Keine erneute Richtlinienumsetzung in Deutschland wegen Streitigkeiten innerhalb der Regierungskoalition}

Die nach Maßgabe der Karlsruher Entscheidungsgründe durchaus mögliche verfassungskonforme Neuregelung der Vorratsdatenspeicherung scheiterte bis zum Ende der Legislaturperiode der CDU/CSU-FDP-Koalition im Herbst 2013 an unüberbrückbaren Meinungsverschiedenheiten zwischen der Bundesjustizministerin Leutheusser-Schnarrenberger (FDP) und dem Bundesinnenminister Friedrich (CSU). Während die Bundesjustizministerin im Interesse eines möglichst weitgehenden Datenschutzes für eine Minimallösung eintrat, befürwortete der Bundesinnenminister im Interesse der effektiven Kriminalitätsbekämpfung eine Maximallösung. Die Wahrung des Koalitionsfriedens stand sowohl einer Kabinettsentscheidung ${ }^{47}$ als auch einem Machtwort der Bundeskanzlerin ${ }^{48}$ in dieser Angelegenheit entgegen. ${ }^{49}$ Wäre die Grundrechtskonformität der Richtlinie durch den EuGH

44 Ibid., Rdnr. 220.

45 BVerfG, Beschl. v. 11.3.2008 (1 BvR 256/08).

46 BVerfG, Beschl. v. 28.10.2008 und 15.10.2009.

47 Art. 65 Satz 3 GG; $\S \S 17,24$ Abs. 2 und 26 Abs. 2 der Geschäftsordnung der Bundesregierung.

48 Art. 65 Satz 1 GG.

49 Im Koalitionsvertrag zwischen CDU, CSU und FDP für die 17. Legislaturperiode v. 26.10.2009, http://www.bmi.bund.de/SharedDocs/Downloads/DE/Ministerium/koalitionsvertrag.pdf?_blo $\mathrm{b}=$ publicationFile (27.1.2014), las man zur Vorratsdatenspeicherung Folgendes: ,Wir werden den Zugriff der Bundesbehörden auf die gespeicherten Vorratsdaten der Telekommunikationsunternehmen bis zur Entscheidung des Bundesverfassungsgerichts über die Verfassungsmäßigkeit der Vorratsdatenspeicherung aussetzen und bis dahin auf Zugriffe zur Abwehr einer konkreten Gefahr für Leib, Leben und Freiheit beschränken." Was nach der Karlsruher Entscheidung geschehen sollte, war nicht geregelt. 
zügig positiv beantwortet worden, hätte die Bundesjustizministerin ihren Widerstand gegen die Umsetzung kaum über Jahre hinweg aufrechterhalten können. Umgekehrt hätte der Bundesinnenminister die Datenzugriffsinteressen des deutschen Sicherheitsapparats nicht hinter der Richtlinie verstecken können, wenn diese wegen Grundrechtsverletzungen vom EuGH zügig für nichtig erklärt worden wäre.

Zur Umsetzung der Vorratsdatenspeicherungs-Richtlinie findet sich nunmehr folgende Passage im neuen Koalitionsvertrag zwischen CDU, CSU und SPD für die 18. Legislaturperiode vom 16. Dezember 2013: „Wir werden die EU-Richtlinie über den Abruf und die Nutzung von Telekommunikationsverbindungsdaten umsetzen. Dadurch vermeiden wir die Verhängung von Zwangsgeldern durch den EuGH. Dabei soll ein Zugriff auf die gespeicherten Daten nur bei schweren Straftaten und nach Genehmigung durch einen Richter sowie zur Abwehr akuter Gefahren für Leib und Leben erfolgen. Die Speicherung der deutschen Telekommunikationsverbindungsdaten, die abgerufen und genutzt werden sollen, haben die Telekommunikationsunternehmen auf Servern in Deutschland vorzunehmen. Auf EU-Ebene werden wir auf eine Verkürzung der Speicherfrist auf drei Monate hinwirken. ${ }^{\text {"50 }}$

Mittlerweile ist ein koalitionsinterner Streit zwischen dem SPD-geführten Bundesjustizministerium und dem CDU-geführten Bundesinnenministerium darüber entstanden, ob vor einem neuen Anlauf zur Umsetzung der Vorratsdatenspeicherungs-Richtlinie in Deutschland die Vorabentscheidung des EuGH in den verbundenen Rechtssachen C-293/12 und C-594/12 abgewartet werden sollte. Während das Bundesinnenministerium auf schnelle Umsetzung drängt, plädiert das Bundesjustizministerium für Geduld. Da zu erwarten ist, dass der EuGH die grundrechtlichen Fragen dieser Vorabentscheidungsersuchen beantworten wird, bevor er die im nächsten Unterabschnitt dargestellte Vertragsverletzungsklage der Kommission gegen Deutschland wegen Nichtumsetzung der Richtlinie entscheidet, spricht mehr für ein Abwarten.

\section{Rechtshängiges Vertragsverletzungsverfahren gegen Deutschland}

Bereits 2011 hatte die Europäische Kommission wegen säumiger Richtlinienumsetzung ein Vertragsverletzungsverfahren nach Art. 258 und Art. 260 Abs. 3 AEUV gegen Deutschland eingeleitet. Am 11. Juni 2012 hat sie dann nach fruchtlosem Vorverfahren Klage beim EuGH erhoben und die Auferlegung eines Zwangsgeldes in Höhe von 315.036,54 Euro pro Tag beantragt. ${ }^{51}$ Auch ohne entsprechenden Antrag der Kommission hat der Gerichtshof die Möglichkeit, neben der Verhängung eines solchen Zwangsgeldes (als Beugezwang zur baldigen Erfüllung der Umsetzungspflicht) Deutschland noch einen Pauschalbetrag (als Sanktion für die jahrelange Untätigkeit des deutschen Gesetzgebers) aufzuerlegen. ${ }^{52}$ Nach seiner ständigen Rechtsprechung kann ein Mitgliedstaat entgegen dem Wortlaut von

50 Koalitionsvertrag zwischen CDU, CSU und SPD, Deutschlands Zukunft gestalten, https:// www.cdu.de/sites/default/files/media/dokumente/koalitionsvertrag.pdf (27.1.2014), S. 147.

51 Anhängig als EuGH, Rs. C-329/12, Europäische Kommission/Bundesrepublik Deutschland. Vgl. EuGH, Rs. C-189/09, Europäische Kommission/Österreich, Slg. 2010, I-99: Verurteilung Österreichs wegen Nichtumsetzung der Richtlinie.

52 EuGH, Rs. C-304/02, Kommission/Frankreich, Slg. 2005, I-6263. 
Art. 277 AEUV den Gerichtshof im Vertragsverletzungsverfahren wegen Nichtumsetzung einer Richtlinie nicht mehr zu einer inzidenten Normenkontrolle veranlassen, wenn er die Klagefrist nach Art. 263 Abs. 6 AEUV versäumt hat. ${ }^{53}$ Diese Rechtsprechung will die Missachtung der Klagefrist des Nichtigkeitsklageverfahrens durch privilegierte Kläger wie die Mitgliedstaaten verhindern: Wenn diese einen Sekundärrechtsakt wegen Verstoßes gegen das Primärrecht für nichtig halten, sollen sie durch Klageerhebung die im rechtsstaatlichen Allgemeininteresse liegende umgehende Klärung durch den EuGH selbst aktiv herbeiführen. Deutschland kann daher im Vertragsverletzungsverfahren nicht mehr geltend machen, die Richtlinie sei wegen Grundrechtsverletzungen nichtig. ${ }^{54}$

Es fragt sich, was aus diesem Vertragsverletzungsverfahren wird, falls der Gerichtshof - den eingangs dargestellten Schlussanträgen des Generalanwalts folgend - die Vorratsdatenspeicherungs-Richtlinie für primärrechtswidrig erklären, ihre Wirkungen (und damit die deutsche Umsetzungsverpflichtung) aber aufrechterhalten sollte. In ständiger Rechtsprechung stellt der EuGH für die Begründetheit einer Klage nach Art. 258 AEUV in Richtlinienfällen darauf ab, ob der beklagte Mitgliedstaat seine Umsetzungsverpflichtung bei Ablauf der Frist vollständig nachgekommen war, die ihm die Kommission in ihrer mit Gründen versehenen Stellungnahme gesetzt hatte. ${ }^{55}$ Dementsprechend könnte Deutschland nur eine (eher unwahrscheinliche) ex tunc wirkende Nichtigerklärung der Richtlinie vor der Verurteilung retten. Zwar stünde es der Kommission frei, im Falle einer anderslautenden Tenorierung seitens des EuGH (etwa im Sinne der vorgenannten Schlussanträge) ihre Klage zurückzunehmen. ${ }^{56}$ Dies ist jedoch nicht zu erwarten, weil sonst eine jahrelange letztlich unberechtigte Vertragsverletzung ungeahndet bliebe. Die Mitgliedstaaten könnten dadurch den Eindruck gewinnen, eine Missachtung von Umsetzungsverpflichtungen lohne sich am Ende doch. ${ }^{57}$

\section{Bestand für das Bundesverfassungsgericht wirklich kein Anlass zur Kooperation mit dem EuGH im Vorabentscheidungsverfahren?}

Es wäre dem Bundesverfassungsgericht ohne weiteres möglich gewesen, die Vereinbarkeit der Vorratsdatenspeicherungs-Richtlinie mit den EU-Grundrechten durch den EuGH definitiv klären zu lassen. Tatsächlich hatten sogar mehrere Beschwerdeführer angeregt, eine Vorabentscheidung gemäß Art. 267 AEUV über die Wirksamkeit der Richtlinie einzuholen. Der Senat hielt jedoch - insoweit einstimmig - ein Vorabentscheidungsersuchen nicht für veranlasst, weil die Wirksamkeit der Richtlinie in den anhängigen Verfassungsbeschwerdeverfahren nicht entscheidungserheblich sei. ${ }^{58}$ Denn diese lasse den mitgliedstaat-

53 Vgl. Schwarze, in: ders. (Hrsg.), EU-Kommentar, 3. Aufl. 2012, Art. 277 AEUV, Rdnr. 6 m.w.N.

54 Kritisch Wunderlich/Hickl, Zum Einwand der Grundrechtswidrigkeit von Richtlinien in Vertragsverletzungsverfahren vor dem Europäischen Gerichtshof, EuR 2013, S. 107 ff.

55 Cremer, in: Calliess/Ruffert, (Fn. 36), Art. 258 AEUV, Rdnr. 33.

56 Vgl. Art. 141 Abs. 1 und 2, Art. 148 EuGH-VerfO.

57 In der Praxis nimmt die Kommission Vertragsverletzungsklagen nur zurück, wenn der beklagte Mitgliedstaat den Vertragsverstoß im Laufe des Verfahrens vollständig abstellt, falls nicht auch in einem solchen Fall noch ein besonderes Interesse an der Feststellung der Vertragsverletzung fortbesteht. Vgl. Wägenbaur, EuGH VerfO, 2008, Art. 78, Rdnr. 4. 
lichen Gesetzgebern einen so großen Entscheidungsspielraum, dass ihre Umsetzung in Deutschland ohne Verstoß gegen die Grundrechte des Grundgesetzes möglich sei. Innerhalb dieses Spielraums könne das Bundesverfassungsgericht das deutsche Umsetzungsgesetz auch uneingeschränkt durch einen etwaigen Vorrang der Richtlinie am Maßstab der deutschen Grundrechte prüfen. Daher hänge der Ausgang der Verfassungsbeschwerdeverfahren nicht von der Wirksamkeit der Richtlinie ab.

Die Karlsruher Begründung ist einerseits plausibel. ${ }^{59}$ Sie verfolgt das verständliche Anliegen, die mit einem Vorabentscheidungsersuchen notwendigerweise verbundenen Verfahrensverzögerungen möglichst zu vermeiden. Dennoch greift sie in ihrer nationalverfassungsrechtlichen Perspektive zu kurz, unterlässt jede europarechtliche Folgenabschätzung, passt nicht zur Europarechtsfreundlichkeit des Grundgesetzes, ${ }^{60}$ begründet Zweifel an der ernsthaften Bereitschaft des Bundesverfassungsgerichts zur Kooperation mit dem EuGH in Grundrechtsfragen und erweist sich nach alledem als Fehlleistung. ${ }^{61}$

Zunächst hat die Nichtigerklärung des Umsetzungsgesetzes bei Fortgeltung der Richtlinie Deutschland automatisch zum Vertragsverletzer gemacht. Dieses wenig europarechtsfreundliche Ergebnis wäre vermeidbar gewesen, wenn das Bundesverfassungsgericht den EuGH durch sein Vorabentscheidungsersuchen von der Nichtigkeit der Richtlinie überzeugt hätte. Ohne Einschaltung des EuGH konnte das Bundesverfassungsgericht auch die nationalen Umsetzungsspielräume gar nicht zuverlässig bestimmen. Denn eine Überprüfung anhand der europäischen Grundrechte hätte den Luxemburger Gerichtshof durchaus $\mathrm{zu}$ einer primärrechtskonformen einengenden Interpretation der Richtlinie veranlassen können, und zwar gerade auch hinsichtlich der Umsetzungsspielräume der Mitgliedstaaten. Dies zeigen die eingangs dargestellten Schlussanträge des Generalanwalts.

Es kommt hinzu, dass ein nationaler Gesetzgeber bei der Umsetzung einer Richtlinie unbestreitbar auch an die EU-Grundrechte gebunden ist, ${ }^{62}$ deren Inhalt in Bezug auf die Vorratsdatenspeicherung der EuGH nicht klären konnte, weil das Bundesverfassungsgericht von einem Vorabentscheidungsersuchen absah. Infolgedessen ist der Bundesgesetzgeber bei seinem überfälligen neuerlichen Versuch, die Vorratsdatenspeicherung im deutschen Recht zu verankern, in dieser Hinsicht auf Mutmaßungen angewiesen. Demgegenüber ist die definitive Feststellung der Wirksamkeit und des grundrechtskonformen Inhalts einer solchen Richtlinie auch für die Klarstellung der demokratischen Verantwortlichkeit unabdingbar: Die nationalen Gesetzgeber müssen erstens sich selbst und zweitens ihren Wählern gegenüber Rechenschaft darüber ablegen, inwieweit sie Freiheitsbeschränkungen noch autonom anordnen können und wollen und inwieweit diese Entscheidung

59 Unplausibel ist allerdings die Formulierung ,etwaiger Vorrang“ in Bezug auf das Verhältnis der Richtlinie zu den deutschen Grundrechten, wie der EuGH im Urteil v. 26.2.2013, Rs. C-399/11, Melloni, Slg. 2013, Rdnr. $55 \mathrm{ff}$. nochmals klargestellt hat.

60 BVerfGE 123, 267 - Lissabon, Rdnrn. 225, 240 f., 340.

61 Vgl. auch Hornung/Schnabel, Verfassungsrechtlich nicht schlechthin verboten - Das Urteil des Bundesverfassungsgerichts in Sachen Vorratsdatenspeicherung, DVB1. 2010, S. 828 f., die den Verdacht äußern, das BVerfG habe seine Formulierung des grundgesetzlich zulässigen Ausmaßes der Vorratsdatenspeicherung an den Vorgaben der Richtlinie orientiert, um eine Vorlage an den EuGH zu vermeiden. Ähnlich Wolff, Vorratsdatenspeicherung - Der Gesetzgeber gefangen zwischen Europarecht und Verfassung?, NVwZ 2010, S. 751.

62 Vgl. Art. 51 Abs. 1 EUGrCh. 
bereits durch den EU-Gesetzgeber getroffen worden ist. Hätte der Bundesgesetzgeber zwischenzeitlich seine Umsetzungsverpflichtung grundgesetzkonform erfüllt und würde der EuGH dann die Richtlinie nachträglich für nichtig erklären, hinge das neue deutsche Umsetzungsgesetz nicht nur politisch und möglicherweise legitimatorisch ,in der Luft“ ${ }^{63}$ Es wäre möglicherweise sogar seinerseits mit denselben europäischen Grundrechten unvereinbar, an denen die zugrundeliegende Richtlinie scheiterte.

Weiterhin dürfen nationale (Verfassungs-)Gerichte ihre Perspektive nicht mehr allein auf den Schutz der Grundrechte der eigenen Bürgerinnen und Bürger beschränken. Vielmehr tragen sie alle eine Mitverantwortung nicht nur für die Entwicklung und Fortbildung, ${ }^{64}$ sondern auch für die inhaltliche Klärung der europäischen Grundrechte im Dialog mit dem EuGH. ${ }^{65}$ Das gilt vor allem in Fällen, in denen Sekundärrechtsakte der EU die Mitgliedstaaten zu Grundrechtseingriffen verpflichten. Hier sind alle nationalen Gerichte aufgerufen, die „Wurzel des Übels“ vom EuGH auf ihre Grundrechtskonformität überprüfen zu lassen. Dem Bundesverfassungsgericht obliegt eine solche Mitverantwortung für das europäische Grundrechtsniveau in besonderem Maße. Denn es hat seit 1974 die Ausbildung europäischer Grundrechte durch seine „Solange“-Rechtsprechung ${ }^{66}$ forciert und ist seit 1992 ein wichtiger Adressat der Struktursicherungsklausel in Art. 23 Abs. 1 Satz 1 GG, die diese Rechtsprechung kodifiziert. Seither steht es in der Pflicht, das in seinen Kräften Stehende zu tun, damit in der EU unter anderen ein dem Grundgesetz im Wesentlichen vergleichbarer Grundrechtsschutz gewährleistet ist. Diese Pflicht muss es durch Kooperation mit dem EuGH im Rahmen des Vorabentscheidungsverfahrens erfüllen und dazu in allen Zweifelsfällen ein Vorabentscheidungsverfahren einleiten, in denen der Gegenstand des bundesverfassungsgerichtlichen Verfahrens mit der europäischen Grundrechtslage eng zusammenhängt. Dies gilt immer, wenn der deutsche Hoheitsakt, um dessen Verfassungsmäßigkeit es geht, zur Durchführung einer europarechtlichen Verpflichtung im Sinne des Art. 51 Abs. 1 Satz 1 EUGrCh ergangen ist.

Schließlich hätte die Vorratsdatenspeicherung - insbesondere im Hinblick auf die oben beschriebene (und kritisierte) Klarstellung des EuGH - dem Bundesverfassungsgericht eine gute Gelegenheit geboten, seine Bereitschaft zur ernsthaften Kooperation mit dem EuGH in Grundrechtsfragen zu dokumentieren. Für ein nationales Höchstgericht, das den EuGH wie kein zweites immer wieder mit Widerstand und Gehorsamsverweigerung bedroht ${ }^{67}$ und zugleich länger als alle anderen mit der Einleitung eines Vorabentscheidungsverfahrens gewartet hat, ist eine entsprechende Geste seit langem überfällig. Der Karlsruher

63 Vgl. sehr weitgehend Payandeh, Die Nichtigkeit von EG-Richtlinien: Konsequenzen für den mitgliedstaatlichen Umsetzungsakt im Lichte des Demokratieprinzips, DVBl. 2007, S. 741 ff.: Bei Nichtigkeit einer Richtlinie verstoße das deutsche Umsetzungsgesetz gegen das Demokratieprinzip des GG und sei daher seinerseits nichtig.

64 Vgl. Art. 6 Abs. 3 EUV, wonach die gemeinsamen Verfassungsüberlieferungen der Mitgliedstaaten eine Erkenntnisquelle für die europäischen Grundrechte bilden.

65 Vgl. Art. 52 Abs. 4 EUGrCh.

66 BVerfGE 37, 271; 73, 339; 102, 147.

67 Vgl. nur BVerfGE 126, 286 - Honeywell. Giegerich, The German Federal Constitutional Court's Misguided Attempts to Guard the European Guardians in Luxemburg and Strasbourg, in: Breuer u.a. (Hrsg.), Der Staat im Recht, Festschrift für Eckart Klein, 2013, S. 49 ff. 
Exzeptionalismus muss auch in Grundrechtsfragen ein Ende nehmen. ${ }^{68}$ Zwar wird es dem durch seine jahrzehntelange unangefochtene Letztinstanzlichkeit selbstbewusst gewordenen Bundesverfassungsgericht besonders schwer fallen, durch ein Vorabentscheidungsersuchen gerade in Bezug auf Grundrechtsfragen die eigene Unterordnung unter die Rechtsprechungsgewalt des EuGH positiv anzuerkennen, denn das vorlegende Gericht ist unstreitig an dessen Vorabentscheidung gebunden. ${ }^{69}$ Andererseits gäbe ein solcher Schritt dem Bundesverfassungsgericht die Möglichkeit, im aktiven Dialog mit dem EuGH dessen Rechtsprechung erheblich zu beeinflussen: Das vorlegende Gericht kann die Vorabentscheidung inhaltlich mitgestalten, wenn es dem EuGH überzeugende Argumente vorträgt. Gerade in Bezug auf das Grundrecht auf Datenschutz - ein Karlsruher Ziehkind ${ }^{70}$ - hätte das Bundesverfassungsgericht auf dem Wege über Art. 267 Abs. 3 AEUV offensiv werden sollen. Sein Wort hätte in dieser Hinsicht in Luxemburg besonderes Gewicht gehabt. Es wäre dann als dasjenige Gericht in die Annalen eingegangen, dem der Datenschutz in der gesamten EU als erstes am Herzen lag. Leider hat es aber den vorbeiwehenden Mantelsaum der europäischen Justizgeschichte verschmäht - ergriffen haben ihn die irischen und österreichischen Richterkolleginnen und -kollegen: Eine bedauerliche Karlsruher Fehlentscheidung.

Über die bisherige grundrechtsschädliche Verzögerungstaktik einer ganzen Reihe Beteiligter tröstet nur ein einziger Umstand ein wenig hinweg: Dass nämlich der EuGH nun just zu einem Zeitpunkt die europäischen Grundrechte auf Privatleben und Datenschutz auslegen und anwenden muss, in dem die europäische Öffentlichkeit deren Wert deutlicher erkennt als je zuvor. Das wiederum verdanken wir dem Whistleblower Edward Snowden, der die unglaublichen Ausspähpraktiken der US-amerikanischen und britischen Geheimdienste publik gemacht hat.

68 Zumindest in Bezug auf das institutionelle Europarecht hat das BVerfG das Eis durch den Beschluss v. 14.1.2014 (2 BvR 2728/13 u.a.) gebrochen. Mit diesem legt es Fragen zur Reichweite der Kompetenzen des Rates der Europäischen Zentralbank dem EuGH zur Vorabentscheidung vor.

69 Wegener, (Fn. 36), Art. 267 AEUV, Rdnr. 47 m.w.N.

70 BVerfGE 65, 1. 